\title{
Literature Overview of the IL-17 Inhibition from Psoriasis to COVID-19
}

\section{Yulin Zou (D) \\ Zudong Meng}

Department of Dermatology, Renmin Hospital, Hubei University of Medicine, Shiyan City, People's Republic of China

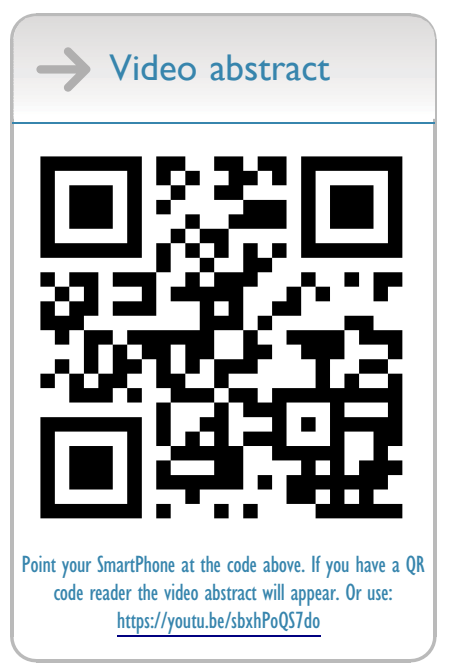

Correspondence: Zudong Meng Email zylmch1007@hbmu.edu.cn

\begin{abstract}
The COVID-19 pandemic has posed a serious problem for drug anti-viral efficacy in combatting the cytokine storm triggered by SARS-CoV-2. From dermato-epidemiological studies conducted on psoriatic and other rheumatological patients, IL-17 inhibitors seem to attenuate or even prevent the cytokine storm and thus ICU referral. Furthermore, both invivo and in-vitro experiments suggest that IL-17 plays a key role in SARS-CoV-2 infection progression. Due to this evidence, we decided to summarize the literature findings on IL-17 inhibitors and COVID-19, maintaining psoriasis as the referral disease to better understand the extent of drug effects on the immune system.
\end{abstract}

Keywords: biologics, COVID-19, psoriasis, therapy, IL-17

\section{Introduction}

Psoriasis (PS) is a chronic inflammatory systemic disease characterized by a variety of comorbidities. PS is also known as a disease characterized by scaling and erythematous plaques. ${ }^{1}$ As a chronic inflammatory disease of the skin, it affects approximately $2 \%$ of the world's population. ${ }^{2}$ As an inflammatory skin disorder it is clinically characterized by scaly and biologically perturbated keratinocytes in a proinflammatory microenvironment. ${ }^{3}$ Psoriasis is a chronic inflammatory papulosquamous disease featuring multiple remissions and relapses. For a long time, it was termed to be primarily a disorder of keratinization. Nowadays, patients often require life-long immunomodulating therapy and no definitive cure exists. ${ }^{4}$ However, the successful use of biological agents in the treatment of psoriasis led to the viewpoint that psoriasis is primarily a disease of IL-23/TH17 cell immune dysregulation. IL-17 inhibitor as one of the most advanced biological agents play an essential role in targeting the IL-23/TH17 pathway. IL-17 inhibitor has changed the treatment for psoriasis. ${ }^{5,6}$

What is also worth noting is that psoriasis patients also develop cardiovascular comorbidities that shorten their lifespan; this aspect is also reflected in the increased cardiovascular burden. ${ }^{7,8}$ According to research by Li et al., pro-coagulative effects are exerted by IL-17 and the antagonization of this cytokine decreases the cardiovascular risk in psoriatic patients. ${ }^{9}$

Meanwhile, the outbreak of severe acute respiratory syndrome coronavirus 2 (SARS-CoV-2) at the end of December 2019 has brought incalculable harm and challenges to more than 200 countries and regions all over the world.

In January 2020 The World Health Organization (WHO) officially termed this disease coronavirus disease 2019 (COVID-19). Meanwhile, the International 
Committee on Taxonomy of Viruses (ICTV) named this new coronavirus severe acute respiratory syndrome coronavirus 2 (SARS-CoV-2). ${ }^{10}$

As is widely known, the disease was initially considered to be an unidentified viral pneumonia. Typical symptoms of COVID-19 are fever, malaise, shortness of breath and even pneumonia, in some severe cases. ${ }^{11}$ A cautionary warning of this disease is that it can be shed through multiple routes. In order to confirm a virus carrier definitively, we need not only molecular but also serological tests. $^{12}$

The more recent research on Covid-19 demonstrates an interface with the the renin-angiotensin-aldosterone system (RAAS) through angiotensin-converting enzyme 2 (ACE2), an enzyme that physiologically counters RAAS activation but also functions as a receptor for SARS viruses. ${ }^{13}$ Moreover, the immune responses to COVID-19 cause T-cell activation and differentiation, including the production of cytokines associated with different T-cell subsets (i.e., Th17), and then release of cytokines in infected cells in response to COVID-19 infection. The pathophysiology for SARS-CoV-2 has not yet been completely understood. It was reported that COVID-19 subjects, particularly those requiring intensive care, have increased concentrations of proinflammatory cytokines (TNF $\alpha$, IL15, and IL17). ${ }^{14}$ In the treatment of psoriasis, as mentioned before, IL-17 is a well-known target of IL-17 inhibitor.

In the early stages of the COVID-19 pandemic, some believed that psoriatic patients undergoing biological treatment, especially with IL-17 inhibitor, can potentially show an increased risk of infection. ${ }^{15}$ However, few articles are available on how the course of COVID-19 infection can influence safety and curative effect of psoriatic patients treated with biological drugs.

Research by Kridin et al. on the impact of immunerelated conditions on the outcomes of COVID-19 found that patients taking biological agents do not influence COVID-19-associated hospitalization or mortality. ${ }^{16}$ However in a current large-scale population-based study, compared with methotrexate and ustekinumab, TNFi treatment during the pandemic was associated with a decreased risk of COVID-19 hospitalization. ${ }^{17}$ An investigation from northern Italy shows that biological therapies during the pandemic cannot influence the development of severe complications of SARS-CoV-2 infection. Similarly there is also no evidence to prove an increased risk of hospitalization or death from COVID-19 in psoriatic patients treated with biologics compared with the general population. ${ }^{18}$ Furthermore, patients treated with biologics may not have an increase in the risk of ICU hospitalization and death. ${ }^{19}$

For IL-17 inhibitors users, a study about IL-17 inhibitors with the risk of COVID-19 Infection, hospitalization, and mortality has proved that IL-17 treatment will not confer an increased risk of COVID-19 infection. The study supports the continuation of IL-17 treatment during the pandemic. ${ }^{20}$

There is real-life evidence on safety and effectiveness from Italian vaccinated healthcare workers. Four cases under biologics who underwent Pfizer mRNABNT162b2 (COMIRNATY) vaccine sustain that vaccines may play a pivotal role in protecting psoriatic patients against SARS-CoV-2 infection. Above all, they do not have to discontinue their prescribed anti-psoriatic therapies at the same time. ${ }^{21}$

As a result, we deem IL-17 inhibitor may be effective for COVID-19 as its treatment in psoriasis.

\section{Immune Response of $\mathbf{T}$ Cells}

Activation of innate and adaptive immune responses can be activated by SARS-CoV-2 infection. Harmful tissue damage may be caused by uncontrolled inflammatory innate responses and impaired adaptive immune responses, both locally and systemically. There is essential evidence that in patients with severe COVID-19, but not in patients with mild disease, lymphopenia is a common feature. Particularly, with drastically reduced numbers of CD4+ $\mathrm{T}$ cells, CD8+ $\mathrm{T}$ cells, B cells and natural killer (NK) cells. In addition, substantially elevated serum levels of pro-inflammatory cytokines exhibit in nearly all patients with severe COVID-19 including IL-17, as well as IL-6 and TNF. ${ }^{22-25}$

A case report of a 50-year-old man who had COVID19 found that the number of peripheral CD4 and CD8 $\mathrm{T}$ cells were substantially reduced from the flow cytometric analysis. Moreover, there was an increased concentration of highly proinflammatory Th17 in CD4 T cells. Additionally, the results imply that overactivation of $\mathrm{T}$ cells, which means increasing of Th17 and high cytotoxicity of CD8 T cells, accounts for, in part, the severe immune injury in the patient. ${ }^{23}$

COVID-19 clinical features are variable and can lead to respiratory and multiorgan failure and systemic manifestations. ${ }^{26}$ Lower leukocyte numbers were shown in COVID-19 patients, as well as abnormal respiratory findings. Th1-Th17 cells increased levels of plasma pro- 
inflammatory cytokines such as interleukin (IL)1- $\beta$, IL1RA, IL6, IL8, IL10. ${ }^{11}$ That is the reason why recently tocilizumab, a monoclonal antibody blocking IL-6 action has been proved to be effective in COVID-19 patients with cytokine storm risk. TCZ appears to be an effective treatment method for COVID-19 patients with a risk of cytokine storms. And for these critically ill patients with elevated IL-6, the repeated use of TCZ is recommended. ${ }^{27}$ Indeed, it can even reduce invasive mechanical ventilation or death in severe COVID-19 pneumonia (defined as the concomitant presence of a respiratory rate $\geq 30$ breaths per minute, blood oxygen saturation $\leq 93 \%$, a $\mathrm{PaO} 2 / \mathrm{FiO} 2$ ratio $<300 \mathrm{mmHg}$ in room air and lung infiltrates $>50 \%$ of the lung, filed within 24-48h).

Particularly, IL-6 in the context of viral infection may be related to a cytokine, which is called IL-17. However, Hou et al. found that the excessive IL-6 level leads to the generation of Th17 cells in murine viral models, and the resulting IL-6 and IL-17 synergistically promote viral constancy by protecting virus infected cells from apoptosis (Figure 1). ${ }^{28}$

In addition, SARS-CoV-2 infection induces psoriatic arthritis flares. Bacterial and viral infectious triggers are linked to psoriatic arthritis (PsA) development. JAK inhibition suppressed activated entheseal plasmacytoid dendritic Type-1 interferon responses as a new novel mechanism of PsA-related arthropathy. Canonical pathway analysis revealed NF-kB signaling, activation of dendritic cell maturation, JAK/STAT signaling pathways and toll-like receptor signaling following oligodeoxynucleotides (ODN) stimulation. SARS-CoV-2 infection is associated with PsA flares. ${ }^{29}$

In response to SARS-CoV-2, antigen-presenting cells (APCs) such as macrophages as well as dendritic cells can present fragments of the antigen to the naive $\mathrm{CD} 4+\mathrm{T}$ cells, and upon activation APCs release TGF- $B$, IL-6, and IL-23 polarizing cytokines. In turn IL-6 binds with its receptor and through JAK-STAT3 causes polarization, maturation, and expansion of CD4+ T cells to Th17 cells with the expression of ROR $\gamma \mathrm{t}$. In turn, the activated Th-17 cells produce inflammatory cytokines such as IL-21, IL-22, IL-17A, and IL-17F.

\section{Why IL-I 7 Inhibitor Can Be Used in COVID-I 9}

COVID-19 has caused a staggering number of cases and deaths. On March 28, 2020, the FDA promulgated an
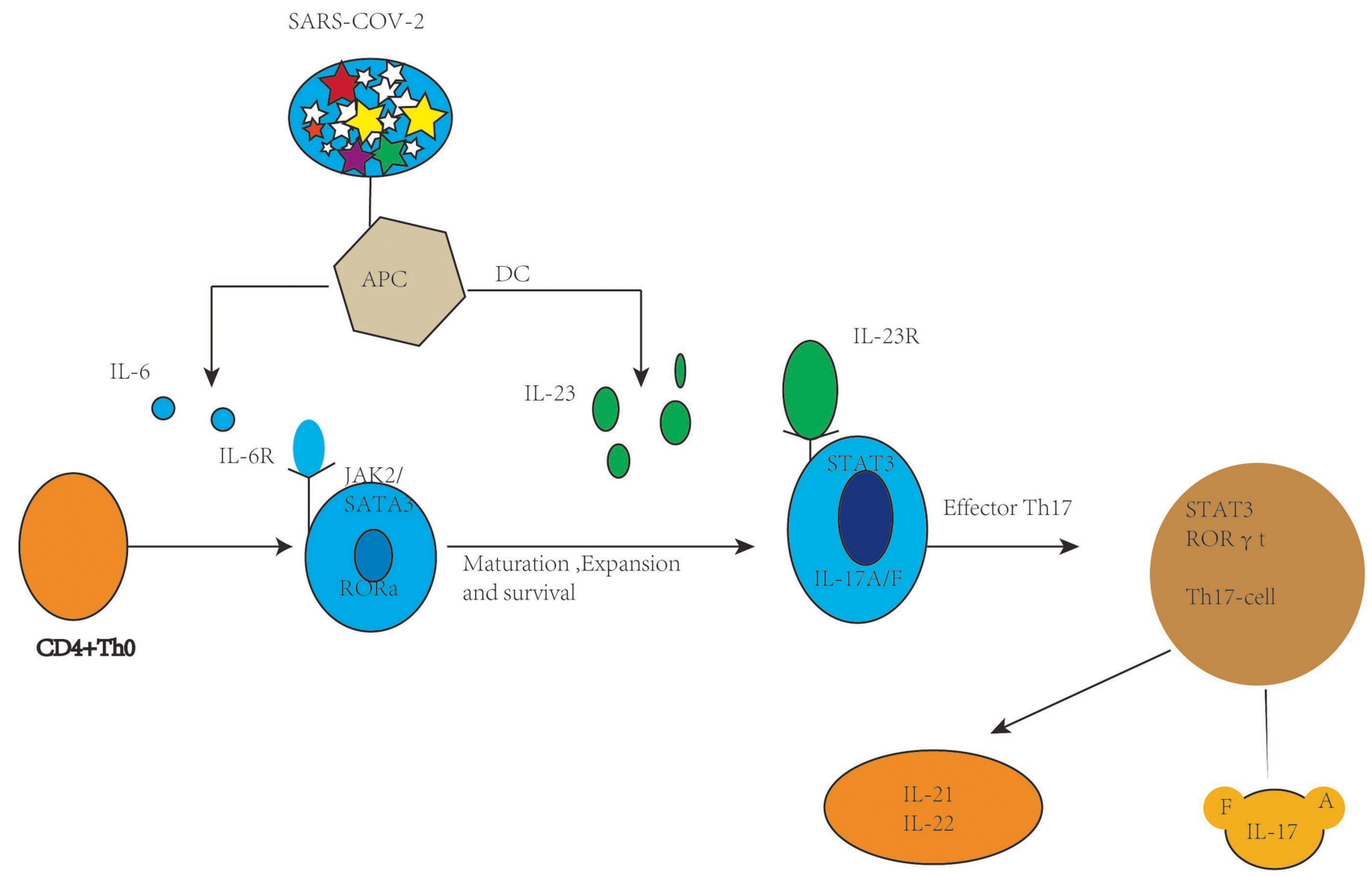

Figure I Th- I7 cell polarization from activation and naive CD4+ T cells. 
emergency use authorisation order for use of chloroquine and hydroxychloroquine in COVID-19 patients, and on May 1, 2020, the FDA gave the same position for use of remdesivir. Now different treatment strategies are undergoing evaluation. However, currently there is no specific treatment available for this disease. ${ }^{30}$ Though trials including remdesivir, hydroxychloroquine, azithromycin and tocilizumab are ongoing in an effort to demonstrate improved outcomes, FDA-approved therapeutics are offlabel investigations.

According the detailed case reported by $\mathrm{Xu}$ et al., the patient's assessment proved an increase in the Th17 subset of CD4+ T cells leading to increased production of IL-17 and IL-22 cytokines that in turn caused the CRS with a rapid and severe deterioration of the patient's condition. A high number of Th17 lymphocytes were visible in alveolar spaces by pathologic postmortem lung analysis. $^{23}$ In addition to the referenced case report, there is an increasing body of evidence supporting that IL-17 plays an important role in the pathogenesis of severe COVID-19 disease, including two recent review articles that further stress the Th17-type cytokine storm in pathogenesis of the disease about immune response in patients with COVID-19. ${ }^{31,32}$ The increase in Th17 CD4+ T cells is not only in COVID-19 but also in several inflammatory diseases, for instance in severe plaque psoriasis. ${ }^{33}$

IL-17 can ligate with IL-17RA, and then initiates activation of transcription factors, such as $\mathrm{NF} \kappa \mathrm{B}, \mathrm{I} \kappa \mathrm{B} \zeta$, API and C-EBP. Later they can potentiate IL-17-induced transcription of the most potent proinflammatory cytokines, even chemokines involved in attracting and further recruitment of immune infiltrates, or matrix metalloproteinases involved in tissue damage. ${ }^{34,35}$

IL-6 levels were remarkably increased on hospitalization days 2 and 3 of the patients, and IL-17 has been demonstrated in blood and affected tissues of patient's hospitalization with COVID-19. ${ }^{23}$ Hence IL-17 inhibitors can block IL-6 and IL-8 secretion from the innate immune system. That could prove a safe and effective therapy for severe COVID-19, as neutrophils, mast cells, $\gamma \delta \mathrm{T}$ cells and innate lymphoid cells (ILC3). There is not only an increased IL-6 level but also increased levels of several proinflammatory Th1, Th2 and Th17 cytokines (e.g., IL-2, $4,6,7,10,12$ and 17) in patients who have severe COVID-19.

IL-17 inhibitor which can block IL-17 alone has been shown as clinically effective in many circumstances and diseases. $^{36,37}$ These broad beneficial opinions support further the idea that IL-17 blocking agents can be tested as potential treatment in severe COVID-19 patients.

\section{Sorts of IL- I 7 Inhibitor}

Although none is yet available for clinical purposes, among the anti-cytokines, monoclonal antibodies are still a potential and possible approach to the prevention of SARS. Anti-IL -17A humanized monoclonal antibodies are well-known and recognized treatment options for psoriasis, such as secukinumab (AIN457) (approved fully human IL-17-specific IgG1k monoclonal antibody) and ixekizumab (LY2439821) (under a Phase III clinical trial for psoriasis). ${ }^{24,38-40}$

Thus far, there have been secukinumab, ixekizumab and brodalumab, three IL-17 blocking agents available. ${ }^{34}$ Secukinumab and ixekizumab have been termed to be very effective in the treatment of severe plaque psoriasis, these being monoclonal IgG1 antibodies that bind specifically to IL-17A. ${ }^{41}$ A quick onset of action, tolerability, high efficacy and a well-established safety profile have been proved in both drugs. Above all, a decrease in the lymphocyte count is not found in those two. ${ }^{34}$ IL-17-targeted biologics are fast acting. Secukinumab was the first IL17A inhibitor that was approved for psoriasis, in 2015. Just a year later, the approval extended to PsA and even ankylosing spondylitis. Patients receiving anti-IL17 biologics secukinumab and ixekizumab have more frequent candida infections (Figure 2). ${ }^{42}$

Brodalumab is a human monoclonal $\operatorname{IgG} 2 \kappa$ antibody against IL-17 receptor A (IL-17RA). Its role is mostly due to complete blockade of key mediators of the T helper 17 pathway, thus inhibiting the biological activity of IL-17A, IL-17F, interleukin-17A/F, and interleukin-17E (also called interleukin-25). ${ }^{43,44}$

\section{Potential Role of IL-I 7 Inhibitor in COVID-I 9 Treatment}

As a global public health problem, COVID-19 has many different treatment approaches which are under investigation and clinical trials are ongoing. Currently, there are no known specific treatment measures for this disease except for meticulous supportive care, for example, mechanical ventilation when indicated. ${ }^{45}$ It is known that the pathological mechanisms most responsible for the COVID-19 death toll are the excessive immune response and inflammatory cell infiltration.

IL-17 inhibitor has been proved in psoriasis treatment, but now they are also utilized in different inflammatory 


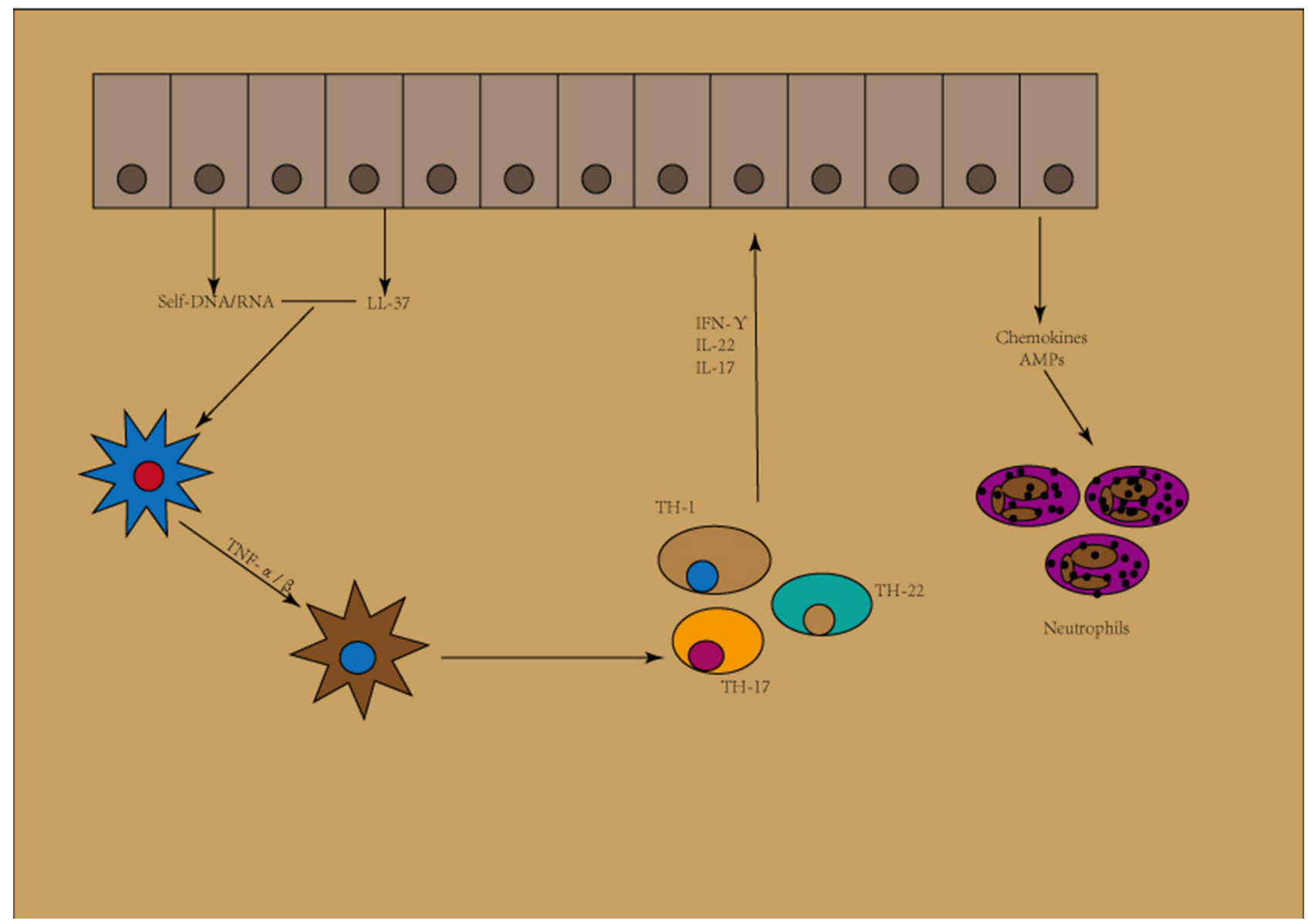

Figure 2 Initiation phase of psoriasis.

diseases, such as ARDS ${ }^{46}$ rheumatism, ${ }^{40}$ and pulmonary fibrosis. ${ }^{47}$ Moreover, respiratory failure in patients presenting with COVID-19 can be attributed to elevated serum IL- $6 .{ }^{48}$ Crowe et al. found in response to pulmonary influenza infection, IL-17RA signalling was important for acute lung injury. This suggests that in treating immunopathology associated with pulmonary viral infections, therapeutic modulation of IL-17 signaling might be useful. ${ }^{49}$ Li et al. demonstrated that acute lung injury is caused by pandemic H1N1 influenza virus in an IL-17-dependent manner in mice. They also reported that IL-17-blocking agents can improve the response to pandemic H1N1 influenza virus in mice. They can also reduce cellular and fluid infiltrates as well as disease duration. IL-17 blocking agents decreased cytokine production by $\mathrm{T}$ cells, the formation of lung edema and pulmonary recruitment of inflammatory cells without preventing virus clearance in mice. $^{50}$

Tocilizumab as a recombinant human monoclonal IgG1 antibody has been shown effective in patients with severe
COVID-19 by blocking its proinflammatory response and IL-6 signaling. ${ }^{51,52}$ Since IL-17 inhibitors and tocilizumab have the same Th17 signaling pathway, IL-17 blocking agents could also be proved as a safe and effective treatment for severe COVID-19. Indeed, IL-17 precedes synthesis of potent proinflammatory TNF- $\alpha$ and cytokines IL-6. Moreover, IL-17 blocking agents could prove more convenient by subcutaneous route of administration rather than tocilizumab, which requires intravenous administration. ${ }^{30}$

\section{Discussion}

Secukinumab is administered subcutaneously in the dose of $300 \mathrm{mg}$ once weekly in the first month of treatment, followed by a dose of $300 \mathrm{mg}$ every 4 weeks in the treatment of severe plaque psoriasis. Ixekizumab is also used $160 \mathrm{mg}$ every 2 weeks during the first 3 months, then followed by a dose of $160 \mathrm{mg}$ every 4 weeks. In the treatment of severe plaque psoriasis, the recommended brodalumab dose is $210 \mathrm{mg}$ once weekly during the first 
2 weeks of treatment, then continued by a dose of $210 \mathrm{mg}$ every 2 weeks administered subcutaneously.

IL-17 inhibitor has not yet been evaluated in clinical trials for COVID-19. However, patients with COVID-19 do not need the same dosage as those with severe plaque psoriasis. The timing of IL-17 inhibitor administration to COVID-19 patients would be crucial as well. We suspect it would perhaps be best to begin in the middle stage of CRS, when an obvious increase in IL-6 levels is to be expected. ${ }^{55}$

Additionally, the deteriorated barrier and inflammatory status could be reversed by IL-17 inhibitor in the skin of psoriasis patients who have less microbe infection. The specific microbe could also be SARS-CoV-2. However, SARS-CoV-2 could stay alive on skin for even $9 \mathrm{~h}$, which may indicate that SARS-CoV-2 might transmit through skin in the certain skin status like psoriasis. Thus, whether the COVID-19 risk could be reduced by IL-17 inhibitor through reversing the inflammatory skin status with deteriorated barrier, and then preventing SARS-CoV-2 transmitting should be further discussed. ${ }^{56,57}$

In our opinion, making use of certain immunomodulators, such as IL-17 inhibitor, might prevent CRS in severe COVID-19 cases, thereby further improving patients' outcomes. Finally, bearing in mind the constraints of limited experimental evidence regarding IL-17 inhibitor, we look forward to an effective and repurposing dosing scheme investigation through conduct of well designed, double blind controlled and randomized clinical trials. IL-17 inhibitor may be a treatment in COVID-19 which appears as a potential promising therapeutic target.

\section{Conclusion}

In this review, we have discussed the current understanding of IL-17 signaling and the underlying immunopathological role as a pro-inflammatory cytokine during SARSCoV-2 infection. According to therapeutic strategies, specific treatments in SARS-CoV-2 infections and related complications still remain challenging. Although there is only limited research evidence, we propose IL-17 inhibitor is a promising therapeutic option to terminate COVID-19, which could limit lung tissue pathology and decrease dangerous cytokines, and help to save lives if used judiciously in appropriate patients.

An increase in IL-17 causes tissue damage and possibly triggers lung edema by contributing to cytokine storm in pulmonary viral infections, including COVID-19. Therefore, IL-17 inhibitor can be good for patients in this regard. As mentioned earlier anti-IL-17 treatments have been found to be beneficial in virus-infected tissues and clinical healing in pandemic influenza infections in mice or humans. At the same time, IL-17 inhibitor apparently can be used in comorbid conditions such as asthma and COPD, which may have infection simultaneously. ${ }^{53}$ Therefore, we believe that IL-17 inhibitor can be successful in people infected with COVID-19.

Artificial Neural Networks (ANNs) allow defining a set of computerized algorithms capable of mimicking and recreating the processes of analysis and learning typical of the human brain. Complex non-linear phenomena, such as biological treatments, have been demonstrated to be more suitable to be evaluated by this approach. This innovative technique to psoriasis patients treated with secukinumab has been proved successful. Hence, these precision medicine based approaches should be extended to all IL17 users, both psoriatic and non-psoriatic patients. ${ }^{54}$

\section{Author Contributions}

Z.D.M. developed the theoretical formalism. Y.L.Z wrote the manuscript with support from Z.D.M. Z.D.M edited and modified the manuscript. All authors made a significant contribution to the work reported, whether that is in the conception, study design, execution, acquisition of data, analysis and interpretation, or in all these areas; took part in drafting, revising or critically reviewing the article; gave final approval of the version to be published; have agreed on the journal to which the article has been submitted; and agree to be accountable for all aspects of the work.

\section{Disclosure}

The authors declare that they have no competing interests.

\section{References}

1. Santus P, Rizzi M, Radovanovic D, et al. Psoriasis and respiratory comorbidities: the added value of fraction of exhaled nitric oxide as a new method to detect, evaluate, and monitor psoriatic systemic involvement and therapeutic efficacy. BioMed Res Int. 2018;2018:3140682. doi:10.1155/2018/3140682

2. Fiore M, Leone S, Maraolo AE, Berti E, Damiani G. Liver Illness and Psoriatic Patients. BioMed Res Int. 2018;2018:3140983. doi:10.1155/ 2018/3140983

3. Damiani G, Bragazzi NL, Garbarino S, et al. Psoriatic and psoriatic arthritis patients with and without jet-lag: does it matter for disease severity scores? Insights and implications from a pilot, prospective study. Chronobiol Int. 2019;36(12):1733-1740. doi:10.1080/ 07420528.2019.1678629

4. Scala E, Megna M, Amerio P, et al. Patients' demographic and socioeconomic characteristics influence the therapeutic decision-making process in psoriasis. PLoS One. 2020;15(8):e0237267. doi:10.1371/ journal.pone. 0237267 
5. Mylle S, Grine L, Speeckaert R, Lambert JLW, van Geel N. Targeting the IL-23/IL-17 Pathway in Psoriasis: the Search for the Good, the Bad and the Ugly. Am J Clin Dermatol. 2018;19(5):625-637. doi:10.1007/s40257-018-0366-5

6. Mahajan R, Handa S. Pathophysiology of psoriasis. Indian J Dermatol Venereol Leprol. 2013;79(Suppl 7):S1-9. doi:10.4103/ 0378-6323.115505

7. Conic RR, Damiani G, Schrom KP, et al. Psoriasis and Psoriatic Arthritis Cardiovascular Disease Endotypes Identified by Red Blood Cell Distribution Width and Mean Platelet Volume. J Clin Med. 2020;9;9(1):E186. doi:10.3390/jcm9010186

8. Seth D, Ehlert AN, Golden JB, et al. Interaction of Resistin and Systolic Blood Pressure in Psoriasis Severity. J Invest Dermatol. 2020;140(6):1279-1282.e1. doi:10.1016/j.jid.2019.07.727

9. Li Y, Golden JB, Camhi MI, et al. Protection from Psoriasis-Related Thrombosis after Inhibition of IL-23 or IL-17A. J Invest Dermatol. 2018;138(2):310-315. doi:10.1016/j.jid.2017.09.021

10. Amerio P, Prignano F, Giuliani F, Gualdi G. COVID-19 and psoriasis: should we fear for patients treated with biologics? Dermatol Ther. 2020;33(4):e13434. doi:10.1111/dth.13434

11. Huang $\mathrm{C}$, Wang $\mathrm{Y}$, Li X, et al. Clinical features of patients infected with 2019 novel coronavirus in Wuhan, China. Lancet Lond Engl. 2020;395(10223):497-506. doi:10.1016/S0140 6736(20)30183-5

12. Zhang W, Du R-H, Li B, et al. Molecular and serological investigation of 2019-nCoV infected patients: implication of multiple shedding routes. Emerg Microbes Infect. 2020;9(1):386-389. doi:10.1080/ 22221751.2020.1729071

13. Vaduganathan M, Vardeny O, Michel T, McMurray JJV, Pfeffer MA, Solomon SD. Renin-Angiotensin-Aldosterone System Inhibitors in Patients with Covid-19. N Engl J Med. 2020;382(17):1653-1659. doi:10.1056/NEJMsr2005760

14. Megna M, Napolitano M, Patruno C, Fabbrocini G. Biologics for psoriasis in COVID-19 era: what do we know? Dermatol Ther. 2020;33(4):e13467. doi:10.1111/dth.13467

15. Conti A, Lasagni C, Bigi L, Pellacani G. Evolution of COVID-19 infection in four psoriatic patients treated with biological drugs. J Eur Acad Dermatol Venereol JEADV. 2020;34(8):e360-1. doi:10.1111/ jdv. 16587

16. Kridin K, Schonmann Y, Tzur Bitan D, et al. Coronavirus Disease 2019 (COVID-19)-Associated Hospitalization and Mortality in Patients with Psoriasis: a Population-Based Study. Am J Clin Dermatol. 2021;22(5):709-718. doi:10.1007/s40257-021-00605-8

17. Kridin K, Schonmann Y, Damiani G, et al. Tumor necrosis factor inhibitors are associated with a decreased risk of COVID-19associated hospitalization in patients with psoriasis-A population-based cohort study. Dermatol Ther. 2021;34(4):e15003. doi:10.1111/dth. 15003

18. Gisondi P, Piaserico S, Naldi L, et al. Incidence rates of hospitalization and death from COVID-19 in patients with psoriasis receiving biological treatment: a Northern Italy experience. J Allergy Clin Immunol. 2021;147(2):558-560.e1. doi:10.1016/j.jaci.2020.10.032

19. Damiani G, Pacifico A, Bragazzi NL, Malagoli P. Biologics increase the risk of SARS-CoV-2 infection and hospitalization, but not ICU admission and death: real-life data from a large cohort during red-zone declaration. Dermatol Ther. 2020;33(5):e13475. doi: $10.1111 /$ dth. 13475

20. Kridin K, Schonmann Y, Solomon A. et al. Risk of COVID-19 Infection, Hospitalization, and Mortality in Patients with Psoriasis Treated by Interleukin-17 Inhibitors. J Dermatol Treat;2021:1-28. doi:10.1080/09546634.2021.1905766

21. Damiani G, Allocco F; Young Dermatologists Italian Network, Malagoli P. COVID-19 vaccination and patients with psoriasis under biologics: real-life evidence on safety and effectiveness from Italian vaccinated healthcare workers. Clin Exp Dermatol. 2021;46 (6):1106-1108. doi:10.1111/ced.14631
22. Huang C, Wang Y, Li X, et al. Clinical features of patients infected with 2019 novel coronavirus in Wuhan, China. Lancet Lond Engl. 2020;395(10223):497-506.

23. $\mathrm{Xu} \mathrm{Z,} \mathrm{Shi} \mathrm{L,} \mathrm{Wang} \mathrm{Y,} \mathrm{et} \mathrm{al.} \mathrm{Pathological} \mathrm{findings} \mathrm{of} \mathrm{COVID-19}$ associated with acute respiratory distress syndrome. Lancet Respir Med. 2020;8(4):420-422. doi:10.1016/S2213-2600(20)30076-X

24. Qin C, Zhou L, Hu Z, et al. Dysregulation of Immune Response in Patients With Coronavirus 2019 (COVID-19) in Wuhan, China. Clin Infect Dis off Publ Infect Dis Soc Am. 2020;71(15):762-768. doi:10.1093/cid/ciaa248

25. Tan M, Liu Y, Zhou R, et al. Immunopathological characteristics of coronavirus disease 2019 cases in Guangzhou, China. Immunology. 2020;160(3):261-268. doi:10.1111/imm.13223

26. Cascella M, Rajnik M, Aleem A, Dulebohn SC, Di Napoli R. Features, Evaluation, and Treatment of Coronavirus (COVID-19). Treasure Island (FL): StatPearls Publishing; 2021. Available from: http://www.ncbi.nlm. nih.gov/books/NBK554776/. Accessed October 18, 2021.

27. Luo P, Liu Y, Qiu L, Liu X, Liu D, Li J. Tocilizumab treatment in COVID-19: a single center experience. J Med Virol. 2020;92 (7):814-818. doi:10.1002/jmv.25801

28. Hou W, Jin Y-H, Kang HS, Kim BS. Interleukin-6 (IL-6) and IL-17 synergistically promote viral persistence by inhibiting cellular apoptosis and cytotoxic T cell function. J Virol. 2014;88(15):8479-8489. doi:10.1128/JVI.00724-14

29. Zhou Q, Vadakekolathu J, Watad A, et al. SARS-CoV-2 Infection Induces Psoriatic Arthritis Flares and Enthesis Resident Plasmacytoid Dendritic Cell Type-1 Interferon Inhibition by JAK Antagonism Offer Novel Spondyloarthritis Pathogenesis Insights. Front Immunol. 2021;12:635018. doi:10.3389/fimmu.2021.635018

30. Bulat V, Situm M, Azdajic MD, Likic R. Potential role of IL-17 blocking agents in the treatment of severe COVID-19? Br J Clin Pharmacol. 2021;87(3):1578-1581. doi:10.1111/bcp.14437

31. Wu D, Yang XO. TH17 responses in cytokine storm of COVID-19: an emerging target of JAK2 inhibitor Fedratinib. $J$ Microbiol Immunol Infect Wei Mian Yu Gan Ran Za Zhi. 2020;53(3):368-370. doi:10.1016/j.jmii.2020.03.005

32. Schett G, Sticherling M, Neurath MF. COVID-19 risk for cytokine targeting in chronic inflammatory diseases? Nat Rev Immunol. 2020;20(5):271-272. doi:10.1038/s41577-020-0312-7

33. Greb JE, Goldminz AM, Elder JT, et al. Psoriasis. Nat Rev Dis Primer. 2016;2:16082. doi:10.1038/nrdp.2016.82

34. Hawkes JE, Chan TC, Krueger JG. Psoriasis pathogenesis and the development of novel targeted immune therapies. J Allergy Clin Immunol. 2017;140(3):645-653. doi:10.1016/j.jaci.2017.07.004

35. Ruiz de Morales JMG, Puig L, Daudén E, et al. Critical role of interleukin (IL)-17 in inflammatory and immune disorders: an updated review of the evidence focusing in controversies. Autoimmun Rev. 2020;19(1):102429. doi:10.1016/j.autrev.2019.102429

36. Mehta P, McAuley DF, Brown M, et al. COVID-19: consider cytokine storm syndromes and immunosuppression. Lancet Lond Engl. 2020;395(10229):1033-1034. doi:10.1016/S0140-6736(20)30628-0

37. Feldmann M, Maini RN, Woody JN, et al. Trials of anti-tumour necrosis factor therapy for COVID-19 are urgently needed. Lancet Lond Engl. 2020;395(10234):1407-1409. doi:10.1016/S01406736(20)30858-8

38. Fletcher JM, Moran B, Petrasca A, Smith CM. IL-17 in inflammatory skin diseases psoriasis and hidradenitis suppurativa. Clin Exp Immunol. 2020;201(2):121-134. doi:10.1111/cei.13449

39. Truchetet M-E, Mossalayi MD, Boniface K. IL-17 in the rheumatologist's line of sight. BioMed Res Int. 2013;2013:295132. doi:10.1155/ 2013/295132

40. Robak E, Gerlicz-Kowalczuk Z, Dziankowska-Bartkowiak B, Wozniacka A, Bogaczewicz J. Serum concentrations of IL-17A, IL-17B, IL-17E and IL-17F in patients with systemic sclerosis. Arch Med Sci AMS. 2019;15(3):706-712. doi:10.5114/ aoms.2019.84738 
41. Wu F, Zhao S, Yu B, et al. A new coronavirus associated with human respiratory disease in China. Nature. 2020;579(7798):265-269. doi:10.1038/s41586-020-2008-3

42. Langley RG, Elewski BE, Lebwohl M, et al. Secukinumab in plaque psoriasis-results of two Phase 3 trials. $N$ Engl J Med. 2014;371 (4):326-338. doi:10.1056/NEJMoa1314258

43. Puig L. Brodalumab: the first anti-IL-17 receptor agent for psoriasis. Drugs Today Barc Spain. 2017;53(5):283-297. doi:10.1358/ dot.2017.53.5.2613690

44. Papp KA, Reich K, Paul C, et al. A prospective Phase III, randomized, double-blind, placebo-controlled study of brodalumab in patients with moderate-to-severe plaque psoriasis. $\mathrm{Br} J$ Dermatol. 2016;175(2):273-286. doi:10.1111/bjd.14493

45. Quartuccio L, Semerano L, Benucci M, Boissier M-C, De Vita S. Urgent avenues in the treatment of COVID-19: targeting downstream inflammation to prevent catastrophic syndrome. Joint Bone Spine. 2020;87(3):191-193. doi:10.1016/j.jbspin.2020.03.011

46. Protective Effects of Anti-IL17 on Acute Lung Injury Induced by LPS in Mice - PubMed [Internet]. [cited Jun 15, 2021]. Available from: https://pubmed.ncbi.nlm.nih.gov/30337870/. Accessed October $18,2021$.

47. Moore JB, June CH. Cytokine release syndrome in severe COVID-19. Science. 2020;368(6490):473-474. doi:10.1126/science. abb8925

48. Georgiev T. Coronavirus disease 2019 (COVID-19) and anti-rheumatic drugs. Rheumatol Int. 2020;40(5):825-826. doi:10.1007/s00296-020-04570-z

49. Crowe CR, Chen K, Pociask DA, et al. Critical role of IL-17RA in immunopathology of influenza infection. J Immunol Baltim Md. 2009;183(8):5301-5310.
50. Li C, Yang P, Sun Y, et al. IL-17 response mediates acute lung injury induced by the 2009 pandemic influenza A (H1N1) virus. Cell Res. 2012;22(3):528-538. doi:10.1038/cr.2011.165

51. Zhang W, Zhao Y, Zhang F, et al. The use of anti-inflammatory drugs in the treatment of people with severe coronavirus disease 2019 (COVID-19): the Perspectives of clinical immunologists from China. Clin Immunol Orlando Fla. 2020;214:108393. doi:10.1016/j. clim.2020.108393

52. Luo P, Liu Y, Qiu L, Liu X, Liu D, Li J. Tocilizumab treatment in COVID-19: a single center experience. J Med Virol. 2020;92 (7):814-818.

53. Ayhan E, Öztürk M, An İ, Abdelmaksoud A, Araç E. Potential role of anti-interleukin-17 in COVID-19 treatment. Dermatol Ther. 2020;33 (4):e13715. doi:10.1111/dth.13715

54. Damiani G, Conic RRZ, Pigatto PDM, et al. Predicting Secukinumab Fast-Responder Profile in Psoriatic Patients: advanced Application of Artificial-Neural-Networks (ANNs). $J$ Drugs Dermatol JDD. 2020;19(12):1241-1246. doi:10.36849/JDD.2020.5006

55. Zhang C, Wu Z, Li J-W, Zhao H, Wang G-Q. Cytokine release syndrome in severe COVID-19: interleukin-6 receptor antagonist tocilizumab may be the key to reduce mortality. Int $J$ Antimicrob Agents. 2020;55(5):105954. doi:10.1016/j.ijantimicag.2020.105954

56. Hirose R, Ikegaya H, Naito Y, et al. Survival of SARS-CoV-2 and influenza virus on the human skin: importance of hand hygiene in COVID-19. Clin Infect Dis off Publ Infect Dis Soc Am. 2020.

57. Xu Q, Chen L, Li X, Zheng J. If skin is a potential host of SARS-CoV-2, IL-17 antibody could reduce the risk of COVID-19. $J \quad$ Am Acad Dermatol. 2021;84(3):e173. doi:10.1016/j. jaad.2020.10.084
Journal of Inflammation Research

\section{Publish your work in this journal}

The Journal of Inflammation Research is an international, peerreviewed open-access journal that welcomes laboratory and clinical findings on the molecular basis, cell biology and pharmacology of inflammation including original research, reviews, symposium reports, hypothesis formation and commentaries on: acute/chronic inflammation; mediators of inflammation; cellular processes; molecular mechanisms; pharmacology and novel anti-inflammatory drugs; clinical conditions involving inflammation. The manuscript management system is completely online and includes a very quick and fair peerreview system. Visit http://www.dovepress.com/testimonials.php to read real quotes from published authors. 